\title{
含叫啶基的单环 $\beta$-内酰胺及噻唑啉酮衍生物的合成及其生物活性
}

\author{
力瓦依丁・买合苏提 ${ }^{a}$ 穆赫塔尔・伊米尔艾山舟 ${ }^{*}$ 麦麦提依明・马合木提 ${ }^{a}$ \\ 萨特瓦尔迪・赫力力 ${ }^{a} \quad$ 刘华君 $^{b}$ \\ ( ${ }^{a}$ 新疆大学化学与化工学院 乌鲁木齐 830046) \\ $\left({ }^{b}\right.$ 新疆农业科学院经济作物研究所 乌鲁木齐 830091)
}

\begin{abstract}
摘要 9-1 $\mathrm{P}$ 啶基甲醛芳亚胺席夫碱分别与氯乙酰氯、芐氧乙酰氯在三乙胺作用下产生的烯酮发生 $[2+2]$ 环加成反应和 颈基乙酸的合环反应，合成了 9 个 1-芳基-3-取代-4-(9-呋啶基)-氮杂环丁-2-酮衍生物 L1 L6 和 2-(9-叫啶基)-3-芳基1,3-塞唑烷-4-酮衍生物 $\mathrm{T} 1 \sim \mathrm{T} 3$, 对所合成的化合物进行了体外抗癌活性和白细胞共同抗原活性篎选. 结构表明, 当样 品浓度为 $10 \mu \mathrm{mol} / \mathrm{L}$ 时，化合物 L4 对肿瘤细胞 HL-60 (Leucocythemia 人白血病细胞)生长的抑制率为 $79.4 \%$. 当样品浓 度为 $20 \mu \mathrm{g} / \mathrm{mL}$ 时, 化合物 L5, L6 和 T3 对细胞分裂周期磷酸醌酶 Cdc25B (Cell division cycle 25B phosphatase)的抑制率 分别为 $80.64 \%, 99.75 \%$ 和 $99.34 \%$. 当样品浓度为 $20 \mu \mathrm{mol} / \mathrm{mL}$ 时，化合物 L6 和 T3 对 CD45 (leukocyte common antigen, LCA 白细胞共同抗原)蛋白酪氨酸磷酸酶 $\mathrm{A}$ 的抑制率分别为 $86.12 \%$ 和 $91.03 \%$. 在此基础上, 初步讨论了该类化合物的 构效关系.
\end{abstract}

关键词 呋啶基; $\beta$-内酰胺; 噻唑啉酮; 合成; 生物活性

\section{Synthesis and Bioactivity of Monocyclic $\beta$-Lactams and 4-Thiazolidinones Derivatives Containing Acridinyl}

\author{
Mahsud, Liwayidin $^{a} \quad$ Imerhasan, Mukhtar $^{*, a} \quad$ Mahmud, Muhammad Amin ${ }^{a}$ \\ Helil, Setiwaldi $^{a} \quad$ Liu, Huajun ${ }^{b}$ \\ $\left({ }^{a}\right.$ College of Chemistry and Chemical Engineering, Xinjiang University, Urumqi 830046) \\ ( ${ }^{b}$ Institute of Industrial Crops, Xinjiang Academy of Agricultural Sciences, Urumqi 830091)
}

\begin{abstract}
In this paper, 1-aryl-3-substituted-4-(9-acridinyl)azetidin-2-one derivatives $\mathbf{L 1} \sim \mathbf{L 6}$ and 2 -(9-acridinyl)3-aryl-1,3-thiazolidin-4-one derivatives $\mathbf{T 1} \sim \mathbf{T 3}$ were synthesized through $[2+2]$ cycloaddition reaction and cyclocondensation reaction of $\mathrm{N}$-( $p$-substitutedaryl)-C-(9-acridinyl)formaldimines Schiff base with ketene, which in situ generated from chloroacetyl chloride and benzyloxyacetyl chloride in the presence of triethylamine, and mercaptoacetic acid. The synthesized compounds have also been screened in vitro anticancer activities and the leukocyte common antigen activities. The results showed that the inhibitory activity of compound $\mathbf{L} 4$ is $79.4 \%$ on human tumor cell HL-60 (Leucocythemia), at the test concentration of $10 \mu \mathrm{mol} / \mathrm{L}$. The inhibitory activities of compounds L5, L6 and T3 on Cdc25B (Cell division cycle 25B) phosphatase are $80.64 \%, 99.75 \%$ and $99.34 \%$ at the test concentration of $20 \mu \mathrm{g} / \mathrm{mL}$, respectively. The inhibitory activities of compounds L6 and T3 on CD45 (leukocyte common antigen, LCA) protein tyrosine phosphatase A are $86.12 \%$ and $91.03 \%$ at the test concentration of $20 \mu \mathrm{mol} / \mathrm{mL}$, respectively. The structure-activity relationship of these compounds was investigated on the bases of bioassay experimental results of these compounds.

Keywords acridinyl; beta-lactam; thiazolidinone; synthesis; biological activity
\end{abstract}

$\beta$-内酰胺类由于其较强的抗菌作用, 在现代医学和 药物研究中被认为不可缺少的抗生素 ${ }^{[1,2]}$, 其在医学上
具有广泛应用价值，而且非对映异构体的 $\beta$-内酰胺类在 有机合成中表现为一种多用的构建模块 ${ }^{[3]}$, 具有抗菌活

\footnotetext{
*E-mail: imerhasan@xju.edu.cn

Received October 7, 2013; revised January 6, 2014; published online February 12, 2014.

Project supported by the Natural Science Foundation of Xinjiang Uyghur Autonomous Region (No. 200421126) and the National Natural Science Foundation of China (No. 21062019).

新疆维吾尔自治区自然科学基金(No. 200421126)、国家自然科学基金(No. 21062019)资助项目.
} 
性的单环 $\beta$-内酰胺抗生素被发现以后, 其作为 $\beta$-内酰胺 类抗生素的重要成员, 该类化合物的设计、合成和立体 化学研究成了研究者们关注的焦点. 噻唑烷-4-酮是一 类广谱的药效基团, 其芳基衍生物多具有抗菌、抗病 毒、抗炎和抗癌等生物活性 ${ }^{[4,5]}$, 叫啶修饰的 $\beta$-内酰胺和 噻唑烷-4-酮是一类具有独特结构的化合物. 由于呋啶 环具有富有电子、空间位阻大、在立体化学选择上可作 为良好取代基团等结构特点, 将叫啶环的引入可以极大 丰富该类化合物的结构多样性, 改善其生物活性. 在与 病菌 DNA 作用较强的药剂研究中呋啶类化合物的研究 目前在国内外是比较活跃. 叫啶类化合物作为 DNA 等 生物大分子的嵌入体 ${ }^{[6,7]}$, 广泛用于抗菌、抗病毒、抗斑 疹伤寒、抗风湿、抗癌、抗肿瘤 ${ }^{[8 \sim 10]}$ 等药物的研发方面. 国内外对于 $\beta$-内酰胺和噻唑啉酮的研究较多, Molteni ${ }^{[11]}$, Krasodomska 等 ${ }^{[12]}$ 研究了席夫碱与烯酮的区域和非对映 选择性，其所带的不同取代基的电子性质、空间位阻等 影响 $\beta$-内酰胺的生物活性. Kumar 等 ${ }^{[13]}$ 研究了单环 $\beta$-内 酰胺体系的合成及其抗病毒活性和细胞毒性评估. Chhajed ${ }^{[14]}$ 和 Sharma 等 ${ }^{[15]}$ 分别研究了含 8-着基喹咥啉和 菜环的单环 $\beta$-内酰胺和噻唑啉酮体系的合成及其抗炎、 抗丝虫作用. 但含呋啶的 $\beta$-内酰胺和噻唑烷-4-酮类化 合物报道较少, 以两个不同骨架的杂环聚集在同一个分 子中后, 观察其对生物活性产生的影响为目的, 本文通 过在三乙胺的作用下氯乙酰氯或芐氧乙酰氯产生的烯 酮与 9-听啶基甲醛芳亚胺席夫碱的 $[2+2]$ 环加成反应, 得到单环 $\beta$-内酰胺类化合物 1-芳基-3-取代-4-(9-呋啶 基)-氮杂环丁-2-酮衍生物 L1 L6; 9-呋啶基甲醛芳亚 胺席夫碱与統基乙酸缩环, 得到噻唑啉酮类化合物 2-(9-呋啶基)-3-芳基-1,3-噻唑烷-4-酮衍生物( T1 T3), 将呋啶环引入到内酰胺或噻唑啉酮环中, 期望由于呋啶 环的引入可改变整个稠杂环分子的空间结构, 引起分子
稳定性、电性的变化，从而增强内酰胺或噻唑啉酩环上 呋啶与细胞 DNA 分子的结合，通过活性篎选，获得高 活性的前提化合物. 合成路线见 Scheme 1.

\section{1 结果与讨论}

\section{1 合成}

所有合成的化合物的结构经 UV-Vis(紫外可见吸收 光谱), ${ }^{1} \mathrm{H} \mathrm{NMR}($ 氢核磁共振谱); IR(红外光谱)和元素分 析表证.

按 Bernthsen 法 ${ }^{[16]}$, 在无水氯化锌的催化下, 二苯 胺和冰乙酸反应得到 9-甲基呋啶. 参照文献[17, 18], 为 了提高亚胺的产率，对 Tsuge 合成亚胺和硝酮的反应进 行改进，将冰乙酸代替盐酸作为缩合试剂，9-甲基呋啶 分别与对亚硝基二甲基苯胺、对亚硝基二乙基苯胺和对 亚硝基苯酚反应，得到相应的原料化合物 $N$-(对取代芳 基)-C-(9-呋啶基)亚胺席夫碱化合物，经重结晶，得到红 棕色固体. 本文按 Staudinger 反应, 在 $-20{ }^{\circ} \mathrm{C}$, 三乙胺 的存在下, 将酰氯的苯溶液滴加到席夫碱苯溶液, 搅拌 回流得到化合物 L1 L6, 化合物 L1, L3, L5 的产率比 化合物 L2, L4, L6 低, 其原因是氯乙酰氯或芐氧乙酰氯, 在三乙胺的作用下, 分别产生氯乙烯酮和芐氧乙烯酮, 由于 $\mathrm{Cl}$ 原子的吸电子能力大于芐氧基氧原子, 正电荷 过于集中于氯乙烯酮 $\alpha-\mathrm{C}$, 使增强氯乙烯酮 $\alpha-\mathrm{C}$ 的正电 性，由此降低了氯乙烯酮的稳定性，部分氯乙烯酮自身 聚合形成二聚体, 减少了与席夫碱环加成的氯乙烯酮. 3 位 $\mathrm{C}$ 和 4 位 $\mathrm{C}$ 是 $[2+2]$ 环加成产物 $\beta(1,3)$-内酰胺结构中 的两个立体化学中心, 也许由此可以产生 4 个非对映异 构体，依据文献顺式异构体中 $3-\mathrm{H}$ 和 $4-\mathrm{H}$ 的偶合常数 $J_{3,4}=5 \mathrm{~Hz}$, 反式异构体中 $3-\mathrm{H}$ 和 4-H 的偶合常数 $J_{3,4}=$ $2.5 \mathrm{~Hz}^{[11,12]}$. 3-H 和 4-H 的偶合常数是判断化合物立体化 学结构是否顺式或反式异构体的主要依据。根据

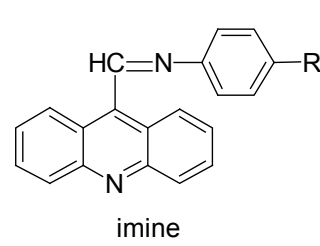

imine

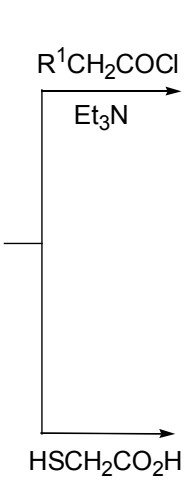

$$
\begin{aligned}
\text { L1 } \mathrm{R} & =\mathrm{N}\left(\mathrm{CH}_{3}\right)_{2}, \mathrm{R}^{1}=\mathrm{Cl} \\
\text { L2 } \mathrm{R} & =\mathrm{N}\left(\mathrm{CH}_{3}\right)_{2}, \mathrm{R}^{1}=\mathrm{OBn} \\
\text { L3 } \mathrm{R} & =\mathrm{N}\left(\mathrm{C}_{2} \mathrm{H}_{5}\right)_{2}, \mathrm{R}^{1}=\mathrm{Cl} \\
\text { L4 R } & =\mathrm{N}\left(\mathrm{C}_{2} \mathrm{H}_{5}\right)_{2}, \mathrm{R}^{1}=\mathrm{OBn} \\
\text { L5 R } & =\mathrm{OH}, \mathrm{R}^{1}=\mathrm{Cl} \\
\text { L6 } \mathrm{R} & =\mathrm{OH}, \mathrm{R}^{1}=\mathrm{OBn}
\end{aligned}
$$

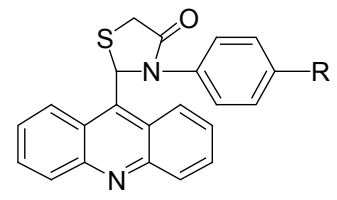

thiazolidinone

T1 $\mathrm{R}=\mathrm{N}\left(\mathrm{CH}_{3}\right)_{2}$, T2 $\mathrm{R}=\mathrm{N}\left(\mathrm{C}_{2} \mathrm{H}_{5}\right)_{2}, \mathbf{T} 3 \mathrm{R}=\mathrm{OH}$

Scheme 1 
${ }^{1} \mathrm{H}$ NMR 谱数据, 化合物 L1, L3, L5 中 $\delta$ 4.95 5.00 和 4.82 4.88 处出现 3 位 $\mathrm{C}$ 和 4 位 $\mathrm{C}$ 质子的吸收峰, 并都 裂分为双峰, 偶合常数 $2.0 \sim 2.2$ 和 $2.1 \sim 2.2 \mathrm{~Hz}, 3$ 位碳上 由于直接与 $\mathrm{C}=\mathrm{O}$ 相连的 $\mathrm{Cl}$ 原子的存在, $3-\mathrm{H}$ 与 $4-\mathrm{H}$ 偶 合而裂分的双峰稍微向低场移, 最多 $\delta 0.05 ; 4$-位碳由于 $N$-(对- $N, N$-二烷基氨基苯基)或 $N$-(对垟基苯基)相连, 4-H 与 3-H 偶合而裂分的双峰稍微向低场移, 最多 $\delta 0.06$; 根据以上数据可判断化合物 $\mathrm{L1}, \mathrm{L3}, \mathrm{L5}$ 的立体化学结构 为反式结构. 化合物 $\mathbf{L 2}, \mathbf{L 4}, \mathbf{L 6}$ 中 $\delta 5.01 \sim 5.04$ 和 4.85 4.89 处出现 3 位 $\mathrm{C}$ 和 4 位 $\mathrm{C}$ 质子的吸收峰, 并都 裂分为双峰, 偶合常数 $5.2 \sim 5.5$ 和 $5.2 \sim 5.4 \mathrm{~Hz}, 3$ 位碳上 由于直接与 $\mathrm{C}=\mathrm{O}$ 相连的 $\mathrm{C}_{6} \mathrm{H}_{5} \mathrm{CH}_{2} \mathrm{O}$ 的存在, $3-\mathrm{H}$ 与 4- $\mathrm{H}$ 偶合而裂分的双峰稍微向低场移, 最多 $\delta 0.03 ; 4$ 位碳由 于 $N$-(对- $N, N$-二烷基氨基苯基)或 $N$-(对羟基苯基)相连, 4-H 与 3-H 偶合而裂分的双峰稍微向低场移, 最多 $\delta$ 0.02; 根据以上数据可判断化合物 $\mathbf{L 2}, \mathbf{L 4}, \mathbf{L 6}$ 的立体化 学结构为顺式结构, 但其绝对构型的判断还需进一步深 入研究.

\section{2 波谱数据分析}

在 ${ }^{1} \mathrm{H}$ NMR 谱中, 化合物 $\mathbf{L 1} \sim \mathbf{L 6}$ 在 $\delta$ 4.95 5.04 和 4.82 4.89 处分别出现了 $\beta(1,3)$-内酰胺环 $3-\mathrm{H}$ 和 $4-\mathrm{H}$ 的双重峰, 偶合常数为 $2.0 \sim 5.5$ 和 $2.1 \sim 5.4 \mathrm{~Hz}$, 在 $\delta$ $7.30 \sim 8.48$ 范围内出现芳环氢的吸收峰, 在 $\delta 3.06$ 和 3.09 处出现了 $\operatorname{ArN}\left(\mathrm{CH}_{3}\right)_{2}$ 中甲基氢的单峰, 在 $\delta 3.42$, 3.46 和 0.98 处分别出现了 $\mathrm{ArN}\left(\mathrm{CH}_{2} \mathrm{CH}_{3}\right)_{2}$ 中亚甲基氢和 甲基氢的四重逢和三重峰, 偶合常数分别为 $7.2 \sim 7.4$ 和 $7.1 \sim 7.2 \mathrm{~Hz}$, 在 $\delta 4.22$ 和 4.45 处出现酚羟基氢的单峰. 在 IR 谱中, 化合物 $\mathbf{L 1} \sim \mathbf{L 6}$ 中 $\mathrm{C}=\mathrm{O}$ 键是 2-氮杂环丁酮 环的特征吸收峰, 在 $1742 \sim 1758 \mathrm{~cm}^{-1}$ 范围内出现了 $v_{\mathrm{C}=\mathrm{O}}$ 的伸缩振动峰, 在 $749 \sim 759 \mathrm{~cm}^{-1}$ 范围内出现了 $v_{\mathrm{C}-\mathrm{Cl}}$ 的吸收峰, 在 $1606 \sim 1644 \mathrm{~cm}^{-1}$ 范围内出现了 $v_{\mathrm{C}=\mathrm{N}}$ 的吸收峰. 当席夫碱的 $\alpha-\mathrm{C}$ 和 $N$-原子分别与呋啶环和 $N$-烷基苯胺等富裕电子效应的基团相连时, 使席夫碱上 电子密度增高; 当烯酮的 $\alpha-\mathrm{C}$ 与 $\mathrm{Cl}$ 或 $\mathrm{C}_{6} \mathrm{H}_{5} \mathrm{CH}_{2} \mathrm{O}$ (芐氧 基)等强吸电基团相连时, 使烯酮的极性增强, 这有利 于烯酮和亚胺的 $[2+2]$ 环加成反应.

化合物 T1 T3 的 ${ }^{1} \mathrm{H}$ NMR 谱中, 在 $\delta \quad 6.31 \sim 6.53$ 处出现 2-位碳原子上一个氢的吸收峰, 噻唑烷酮中 2-位 碳原子由于与 $\mathrm{N}$ 和 $\mathrm{S}$ 等吸电子原子相连, 致使 $\mathrm{C}-2$ 质子 的吸收峰向低场移动, 在 $\delta \quad 4.20 \sim 4.58$ 和 $3.37 \sim 3.59$ 处 出现 5 -位碳原子上氢的双重峰, 偶合常数为 $15.2 \sim 15.6$ $\mathrm{Hz}$, 在 $\delta 6.68 \sim 8.27$ 范围内出现芳环氢的吸收峰, 在 $\delta$ 3.06 和 3.07 处出现了 $\mathrm{ArN}\left(\mathrm{CH}_{3}\right)_{2}$ 中甲基氢的单峰, 在 $\delta$ 3.14 和 0.99 处分别出现了 $\mathrm{ArN}\left(\mathrm{CH}_{2} \mathrm{CH}_{3}\right)_{2}$ 中亚甲基氢和 甲基氢的四重逢和三重峰, 偶合常数分别为 6.8 和 7.2
$\mathrm{Hz}$, 在 $\delta 3.93$ 处出现酚羟基氢的单峰. IR 谱中, 化合物 $\mathbf{T 1} \sim \mathbf{T} 3$ 中 $\mathrm{C}=\mathrm{O}$ 键和 $\mathrm{C}-\mathrm{S}-\mathrm{C}$ 是 1,3-噻唑烷-4-酮环的 特征吸收峰, 在 1703 1709 $\mathrm{cm}^{-1}$ 范围内出现了 $v_{\mathrm{C}=\mathrm{O}}$ 的 强伸缩振动峰, 在 $674 \sim 687 \mathrm{~cm}^{-1}$ 范围内出现 $v_{\mathrm{C}^{-} \mathrm{S}-\mathrm{C}}$ 的 中强伸缩振动峰, 在 $1358 \sim 1367 \mathrm{~cm}^{-1}$ 范围内出现了 $v_{\mathrm{C}-\mathrm{N}}$ 的中强伸缩振动峰, 在 $1602 \sim 1611 \mathrm{~cm}^{-1}$ 范围内出 现了 $v_{\mathrm{C}=\mathrm{N}}$ 的吸收峰.

\section{3 生物活性和构效关系}

经国家新药筛选中心对化合物 L1 L6 进行了对体 外培养人白血病细胞株、对抗癌药物靶点细胞分裂周期 磷酸酯酶 $\mathrm{Cdc} 25 \mathrm{~B}$ (或 Cdc25A)和 CD45 蛋白酪氨酸磷酸 酶 $\mathrm{A}$ 抑制活性测试, 结果表明, 当样品浓度为 10 $\mu \mathrm{mol} / \mathrm{L}$ 时, 化合物 $\mathbf{L} 4$ 对肿瘤细胞 HL-60(人白血病细胞) 生长的抑制率为 $79.4 \%$. 当样品浓度为 $20 \mu \mathrm{g} / \mathrm{mL}$ 时, 化 合物 L5, L6 和 T3 对细胞分裂周期磷酸酯酶 Cdc25B 的 抑制率分别为 $80.64 \%, 99.75 \%$ 和 $99.34 \%$. 当样品浓度为 $20 \mu \mathrm{mol} / \mathrm{mL}$ 时, 化合物 L6 和 T3 对 CD45 蛋白酪氨酸 磷酸酶 $\mathrm{A}$ 的抑制率分别为 $86.12 \%$ 和 $91.03 \%$. 其余化合 物对 $\mathrm{Cdc} 25 \mathrm{~B}$ (或 $\mathrm{Cdc} 25 \mathrm{~A}$ )酶、CD45 蛋白酪氨酸磷酸酶 $\mathrm{A}$ 和对 HL-60 细胞株生长的抑制率 $\left(\mathrm{IC}_{50}\right.$ 值)都小于 $50 \%$. 根据生物活性测试结果, 可得出: 化合物 $\mathbf{L 1} \sim \mathbf{L 6}$ 和 T1 T3 中, $\beta$-内酰胺环和噻唑啉酮环的不同取代基影响 它们的生物活性. 其生物活性取决于取代基的富有电子 性质、空间位阻和氢键性等诸多因素. 如杂环取代基引 入到 $\beta$-内酰胺环的 3 位 $\mathrm{C}$ 或 4 位 $\mathrm{C}$ 时, 它表现的生物活 性增大人们对这类化合物的研究兴趣. 相同浓度下，同 一个测试模型中测试结果表明, 化合物 L6 对 Cdc25B 酶 和 CD45 酶的抑制率高于 $\mathbf{L 5}$, 化合物 $\mathbf{L} \mathbf{4}$ 对肿瘤细胞 HL-60 生长的抑制率高于 L1, 化合物 $\mathbf{T 3}$ 对 Cdc25B 酶 的抑制率高于 $\mathbf{T} 2$ 和 $\mathbf{T} 1$. 这是因为芳基、听啶杂环以及 氯或芐氧基等不同的取代基同时聚集在 $\beta$-内酰胺环时, 一方面由于 $\mathrm{Cl}$ 原子的电负性大于芐氧基氧原子, 使 $\mathrm{Cl}$ 原子比芐氧基降低了 $\beta$-内酰胺环的电子密度. 另一方面 在 $\beta$-内酰胺环 (1 位 $\mathrm{C}$ 和 4 位 $\mathrm{C}$ )或噻唑啉酮环 ( 2 位 $\mathrm{C}$ 和 3 位 C)中由于相邻两个 $\mathrm{C}$ 原子分别与对羟基苯基和呋啶 杂环等富有电子体系相连, 其对内酰胺环或噻唑啉酮环 具有电子诱导效应，另外它们之间的空间位阻，使它们 相互处于环平面的不同面, 呋啶环被更加露出, 这就有 利于增强呋啶与细胞 DNA 之间的作用力, 由此提高了 $\beta$-内酰胺或噻唑啉酮的药效.

\section{2 结论}

根据活性亚结构拼接原理,本文以特定结构功能的 呋啶环修饰 $\beta$-内酰胺和噻唑烷-4-酮设计合成目标化合 物 1-芳基-3-取代-4-(9-呋啶基)-氮杂环丁-2-酮衍生物 
L1 L6 和 2-(9-呋啶基)-3-芳基-1,3-噻唑烷-4-酮衍生物 $\mathbf{T 1} \sim \mathbf{T 3}$, 其合成操作简便、易后处理等特点. 所合成的 目标化合物中化合物 L4, L5, L6 和 T3 显示了较强的抗 癌和抗炎症性及免疫性疾病活性.

\section{3 实验部分}

\section{1 仪器与试剂}

熔点仪用 X-6 精密显微熔点测定仪(温度计未经校 正); 紫外分光光度计 UV-2450; Varian Inova-400 型核磁 共振仪(TMS 为内标, $\mathrm{CDCl}_{3}, \mathrm{CD}_{3} \mathrm{OD}$ 或 DMSO- $d_{6}$ 作溶 剂); BRUKER EQINOX55 型红外光谱仪( $\mathrm{KBr}$ 压片); Perkin-Elmer 2400CHN 型元素分析仪.

三乙胺和溶剂用前重蒸处理, 其它试剂均为市售分 析纯, 用前未经处理; $N$-(对取代芳基)-C-(9-呋啶基)亚胺 参照文献 $[17,18]$ 制备.

\subsection{1-芳基-3-取代-4-(9-叫啶基)-氮杂环丁-2-酮衍生 物 $\mathrm{L} 1 \sim \mathrm{L} 6$ 的合成}

以 1-(对- $N, N$-二甲基氨基苯基)-3-氯-4-(9-呋啶基)氮杂环丁-2-酮 $(\mathbf{L} 1)$ 的合成为例. 在带干燥装置和通氮气 的 $100 \mathrm{~mL}$ 的三口瓶中放入 $1.56 \mathrm{mmol} N$-(对 $-N, N$-二甲氨 基苯基)-9-呋啶甲醛亚胺和 $10 \mathrm{~mL}$ 苯(新蒸馏), 油浴缓慢 加热和磁力摚拌下滴加 $4.68 \mathrm{~mol}$ (3 equiv.)三乙胺的 5 $\mathrm{mL}$ 苯溶液. 然后反应液冷却至 $-20{ }^{\circ} \mathrm{C}$ 、并搅拌下缓慢 滴加 $3.12 \mathrm{mmol}$ (2 equiv.)氯乙酰氯的 $10 \mathrm{~mL}$ 苯溶液(需 $1 \sim 1.5 \mathrm{~h})$. 移去冷却浴, 缓慢升温后, 在油浴继续摚拌 回流 15 24 h. 溶液颜色变深, TLC 检测反应. 反应结 束后, 反应液冷至室温, 依此用 $50 \mathrm{~mL} \mathrm{NaHCO}_{3}$ 饱和溶 液和 $50 \mathrm{~mL} \mathrm{NaCl}$ 饱和溶液洗涤, 分出有机层, 用无水 $\mathrm{MgSO}_{4}$ 干燥、过滤、浓缩旋干溶剂, 真空干燥, 得到黑 绿色粘状固体. 固体用少量 $\mathrm{CHCl}_{3}$ 溶解后, 柱层析 $\left[\mathrm{SiO}_{2}\right.$, 洗脱剂: $V$ (石油醚) $: V$ (乙酸乙酯 $\left.)=6: 4\right]$ 纯化, 收集 $R_{\mathrm{f}}=0.50 \sim 0.64$ 的斑点, 得 $\mathbf{L 1} \sim \mathbf{L 6}$.

L1：黑绿色固体, $167.8 \mathrm{mg}$, 产率 $29.9 \%$. m.p 177 $178{ }^{\circ} \mathrm{C}$; UV-Vis $\left(\mathrm{CHCl}_{3}\right) \lambda_{\max }: 256,384 \mathrm{~nm} ;{ }^{1} \mathrm{H}$ NMR $\left(\mathrm{CDCl}_{3}, 400 \mathrm{MHz}\right) \delta: 7.53 \sim 8.39(\mathrm{~m}, 12 \mathrm{H}, \mathrm{ArH}), 4.98$ (d, $\left.J_{3,4}=2.2 \mathrm{~Hz}, 1 \mathrm{H}, 3-\mathrm{H}\right), 4.82\left(\mathrm{~d}, J_{4,3}=2.1 \mathrm{~Hz}, 1 \mathrm{H}, 4-\mathrm{H}\right)$, 3.06, 3.09 [s, 6H, N(CH$\left.)_{2}\right]$; IR $(\mathrm{KBr}) v$ : $3061(\mathrm{Ar}-\mathrm{H})$, 2924, $2853(\mathrm{C}-\mathrm{H}), 1754(\mathrm{C}-\mathrm{N}=\mathrm{O}), 1606(\mathrm{C}=\mathrm{N})$, 1514, 1463, $1365(\mathrm{C}-\mathrm{N}), 749(\mathrm{C}-\mathrm{Cl}), 698 \mathrm{~cm}^{-1}$. Anal. calcd for $\mathrm{C}_{24} \mathrm{H}_{20} \mathrm{~N}_{3} \mathrm{OCl}$ : C 71.73, H 5.02, N 10.46; found $\mathrm{C}$ 71.81, H 5.12, N 10.79 .

L2: 棕红色固体, $156.7 \mathrm{mg}$, 产率 36.8\%. m.p. 222 $223{ }^{\circ} \mathrm{C}$; UV-Vis $\left(\mathrm{CHCl}_{3}\right) \lambda_{\max }: 290,396 \mathrm{~nm} ;{ }^{1} \mathrm{H}$ NMR (400 $\left.\mathrm{MHz}, \mathrm{CDCl}_{3}\right) \delta: 7.51 \sim 8.47(\mathrm{~m}, 17 \mathrm{H}, \mathrm{Ar}-\mathrm{H}), 5.03(\mathrm{~d}$, $\left.J_{3,4}=5.4 \mathrm{~Hz}, 1 \mathrm{H}, 3-\mathrm{H}\right), 4.85\left(\mathrm{~d}, J_{4,3}=5.2 \mathrm{~Hz}, 1 \mathrm{H}, 4-\mathrm{H}\right)$, $4.63\left(\mathrm{~d}, J=11.2 \mathrm{~Hz}, 1 \mathrm{H}, \mathrm{OCH}_{2} \mathrm{Ph}\right), 4.54(\mathrm{~d}, J=11.2 \mathrm{~Hz}$, $\left.1 \mathrm{H}, \mathrm{OCH}_{2} \mathrm{Ph}\right), 3.06,3.09$ [s, 6H, N($\left.\left(\mathrm{CH}_{3}\right)_{2}\right]$; IR $(\mathrm{KBr}) v$ : $3062(\mathrm{Ar}-\mathrm{H}), 2924,2854(\mathrm{C}-\mathrm{H}), 1745(\mathrm{~N}-\mathrm{C}=\mathrm{O})$, $1609(\mathrm{C}=\mathrm{N}), 1599,1513,1462,1371(\mathrm{C}-\mathrm{N}), 1246$, 1196, 1029, 794, $699 \mathrm{~cm}^{-1}$. Anal. calcd for $\mathrm{C}_{31} \mathrm{H}_{27} \mathrm{~N}_{3} \mathrm{O}_{2}$ : C 78.62, H 5.75, N 8.87; found C 78.73, H 5.78, N 8.79.

L3: 棕黑色固体, $142.6 \mathrm{mg}$, 产率 33.2\%. m.p. 182 $183{ }^{\circ} \mathrm{C}$; UV-Vis $\left(\mathrm{CHCl}_{3}\right) \lambda_{\max }: 296,385 \mathrm{~nm} ;{ }^{1} \mathrm{H}$ NMR (400 $\left.\mathrm{MHz}, \mathrm{CDCl}_{3}\right) \delta: 7.32 \sim 8.48(\mathrm{~m}, 12 \mathrm{H}, \mathrm{Ar}-\mathrm{H}), 4.95(\mathrm{~d}$, $\left.J_{3,4}=2.0 \mathrm{~Hz}, 1 \mathrm{H}, 3-\mathrm{H}\right), 4.84\left(\mathrm{~d}, J_{4,3}=2.2 \mathrm{~Hz}, 1 \mathrm{H}, 4-\mathrm{H}\right)$, 3.42 (q, $J=7.2 \mathrm{~Hz}, 4 \mathrm{H}, \mathrm{NCH}_{2} \mathrm{CH}_{3}$ ), 0.98 (t, $J=7.2 \mathrm{~Hz}$, $\left.6 \mathrm{H}, \mathrm{NCH}_{2} \mathrm{CH}_{3}\right)$; IR (KBr) v: $3055(\mathrm{Ar}-\mathrm{H}), 2957,2857$ $(\mathrm{C}-\mathrm{H}), 1752(\mathrm{~N}-\mathrm{C}=\mathrm{O}), 1608(\mathrm{C}=\mathrm{N}), 1516,1461(\mathrm{C}=$ C), $1351(\mathrm{C}-\mathrm{N}), 752(\mathrm{C}-\mathrm{Cl}), 698 \mathrm{~cm}^{-1}$. Anal. calcd for $\mathrm{C}_{26} \mathrm{H}_{24} \mathrm{~N}_{3} \mathrm{OCl}$ : C 72.63, H 5.63, N 9.77; found C 72.69, H 5.57, N 9.89 .

L4: 棕红色固体, $154.7 \mathrm{mg}$, 产率 41.2\%. m.p. 112 $114{ }^{\circ} \mathrm{C}$; UV-Vis $\left(\mathrm{CHCl}_{3}\right) \lambda_{\max }: 296,410 \mathrm{~nm} ;{ }^{1} \mathrm{H}$ NMR (400 $\left.\mathrm{MHz}, \mathrm{CDCl}_{3}\right) \delta: 7.52 \sim 8.45(\mathrm{~m}, 17 \mathrm{H}, \operatorname{Ar}-\mathrm{H}), 5.01(\mathrm{~d}$, $\left.J_{3,4}=5.2 \mathrm{~Hz}, 3-\mathrm{H}\right), 4.86\left(\mathrm{~d}, J_{4,3}=5.2 \mathrm{~Hz}, 1 \mathrm{H}, 4-\mathrm{H}\right), 4.59$ (d, $\left.J=11.4 \mathrm{~Hz}, 1 \mathrm{H}, \mathrm{OCH}_{2} \mathrm{Ph}\right), 4.48(\mathrm{~d}, J=11.4 \mathrm{~Hz}, 1 \mathrm{H}$, $\mathrm{OCH}_{2} \mathrm{Ph}$ ), 3.46 (q, $J=7.4 \mathrm{~Hz}, 4 \mathrm{H}, \mathrm{NCH}_{2} \mathrm{CH}_{3}$ ), 0.98 (t, $J=$ $\left.7.1 \mathrm{~Hz}, 6 \mathrm{H}, \mathrm{NCH}_{2} \mathrm{CH}_{3}\right)$; IR (KBr) v: $3046(\mathrm{Ar}-\mathrm{H}), 2959$, $2852(\mathrm{C}-\mathrm{H}), 1742(\mathrm{~N}-\mathrm{C}=\mathrm{O}), 1614(\mathrm{C}=\mathrm{N}), 1583,1451$ $(\mathrm{C}=\mathrm{C}), 1355(\mathrm{C}-\mathrm{N})$ 1258, 1156, 1071, 759, $696 \mathrm{~cm}^{-1}$. Anal. calcd for $\mathrm{C}_{33} \mathrm{H}_{31} \mathrm{~N}_{3} \mathrm{O}_{2}$ : C 79.01, $\mathrm{H}$ 6.23, N 8.38; found C 79.17, H 6.19, N 8.29.

L5: 黑绿色固体, $192.6 \mathrm{mg}$, 产率 34.3\%. m.p. 241 $242{ }^{\circ} \mathrm{C}$; UV-Vis $\left(\mathrm{CHCl}_{3}\right) \lambda_{\max }: 291,388 \mathrm{~nm} ;{ }^{1} \mathrm{H}$ NMR (400 $\left.\mathrm{MHz}, \mathrm{CD}_{3} \mathrm{OD}\right) \delta: 7.40 \sim 8.34(\mathrm{~m}, 12 \mathrm{H}, \mathrm{Ar}-\mathrm{H}), 5.00(\mathrm{~d}$, $\left.J_{3,4}=2.2 \mathrm{~Hz}, 1 \mathrm{H}, 3-\mathrm{H}\right), 4.88\left(\mathrm{~d}, J_{4,3}=2.2 \mathrm{~Hz}, 1 \mathrm{H}, 4-\mathrm{H}\right)$, 4.45 (s, IH, OH); IR (KBr) v: $3329(\mathrm{O}-\mathrm{H}), 3039(\mathrm{Ar}-\mathrm{H})$, 2976, $2854(\mathrm{C}-\mathrm{H}), 1758(\mathrm{~N}-\mathrm{C}=\mathrm{O}), 1644(\mathrm{C}=\mathrm{N}), 1493$ $(\mathrm{C}=\mathrm{C}), 1385(\mathrm{C}-\mathrm{N}), 1169,1071,759(\mathrm{C}-\mathrm{CI}), 675 \mathrm{~cm}^{-1}$. Anal. calcd for $\mathrm{C}_{22} \mathrm{H}_{15} \mathrm{~N}_{2} \mathrm{O}_{2} \mathrm{Cl}$ : C 70.50, H 4.03, N 7.47; found C 70.47, H 4.01, N 7.54.

L6: 棕黑色固体, $175.3 \mathrm{mg}$ ，产率 36.2\%. m.p. 175 $176{ }^{\circ} \mathrm{C}$; UV-Vis $\left(\mathrm{CHCl}_{3}\right) \lambda_{\max }: 290,357 \mathrm{~nm} ;{ }^{1} \mathrm{H}$ NMR (400 $\left.\mathrm{MHz}, \mathrm{DMSO}-d_{6}\right) \delta: 7.30 \sim 8.44(\mathrm{~m}, 12 \mathrm{H}, \mathrm{Ar}-\mathrm{H}), 5.04(\mathrm{~d}$, $\left.J_{3,4}=5.5 \mathrm{~Hz}, 1 \mathrm{H}, 3-\mathrm{H}\right), 4.89\left(\mathrm{~d}, J_{4,3}=5.3 \mathrm{~Hz}, 1 \mathrm{H}, 4-\mathrm{H}\right)$, 4.68 (d, $\left.J=11.7 \mathrm{~Hz}, 1 \mathrm{H}, \mathrm{OCH}_{2} \mathrm{Ph}\right), 4.57$ (d, $J=11.7 \mathrm{~Hz}$, $\left.1 \mathrm{H}, \mathrm{OCH}_{2} \mathrm{Ph}\right), 4.22$ (s, 1H, OH); IR (KBr) v: $3435(\mathrm{O}-\mathrm{H})$, $3059(\mathrm{Ar}-\mathrm{H}), 2979,2884(\mathrm{C}-\mathrm{H}), 1748(\mathrm{C}=0), 1635$ $(\mathrm{C}=\mathrm{N}), 1478(\mathrm{C}=\mathrm{C}), 1380(\mathrm{C}-\mathrm{N}), 1152,1073,742$ $(\mathrm{C}-\mathrm{CI}), 678 \mathrm{~cm}^{-1}$. Anal. calcd for $\mathrm{C}_{29} \mathrm{H}_{22} \mathrm{~N}_{2} \mathrm{O}_{3}: \mathrm{C} 78.01$, 
H 4.97, N 6.27; found C 77.93, H 4.87, N 6.33.

\subsection{2-(9-叫啶基)-3-芳基-1,3-噻唑烷-4-酮衍生物 (T1 T T3)的合成}

2-(9-呋啶基)-3-(对二甲氨基苯基)-1,3-噻唑烷-4-酤 (T3)的合成为例. 在 $50 \mathrm{~mL}$ 圆底烧瓶中置于 $3.11 \mathrm{mmol}$ $N$-(对- $N, N$-二甲氨基苯基)-9-呋啶甲醛亚胺和 $3.42 \mathrm{mmol}$ (1.1 equiv.)疏基乙酸溶于 $25 \mathrm{~mL}$ 苯(新蒸馏)中, 在磁力 摚拌下, 油浴加热回流 $12 \sim 18 \mathrm{~h}$, 反应中产生的水用水 分离器分离, TLC 检测反应, 反应结束后, 减压蒸干溶 剂, 反应液冷至室温, 加 $40 \mathrm{~mL}, 20 \%$ 热 $\mathrm{Na}_{2} \mathrm{CO}_{3}$ 溶液得 到棕黑色悬浮液, 过滤得棕黑色粘状固体, 用乙醇多次 重结晶, 得 $\mathbf{T} 1 \sim \mathbf{T} 3$.

T1：棕红色粉末状固体, $215.0 \mathrm{mg}$, 产率 $37.5 \%$. m.p. $151 \sim 152{ }^{\circ} \mathrm{C}$; UV-Vis $\left(\mathrm{CHCl}_{3}\right) \lambda_{\max }: 251,288 \mathrm{~nm}$; ${ }^{1} \mathrm{H}$ NMR (400 MHz, $\left.\mathrm{CDCl}_{3}\right) \delta: 6.86 \sim 7.72(\mathrm{~m}, 12 \mathrm{H}, \mathrm{ArH})$, $6.36(\mathrm{~s}, 1 \mathrm{H}, 2-\mathrm{H}), 4.20$ (d, $\left.J_{5 \mathrm{a}, 5 \mathrm{~b}}=15.6 \mathrm{~Hz}, 1 \mathrm{H}, 5-\mathrm{H}_{\mathrm{a}}\right), 3.59$ $\left(\mathrm{d}, J_{5 \mathrm{~b}, 5 \mathrm{a}}=15.6 \mathrm{~Hz}, 1 \mathrm{H}, 5-\mathrm{H}_{\mathrm{b}}\right), 3.06,3.07$ [s, 6H, N($\left.\left(\mathrm{CH}_{3}\right)_{2}\right]$; IR (KBr) v: $3046(\mathrm{Ar}-\mathrm{H}), 2980,2886(\mathrm{C}-\mathrm{H}), 1706(\mathrm{C}=$ O), $1604(\mathrm{C}=\mathrm{N}), 1583,1514,1480(\mathrm{C}=\mathrm{C}), 1359(\mathrm{C}-\mathrm{N})$, 1136, 1067, $678(\mathrm{C}-\mathrm{S}-\mathrm{C}) \mathrm{cm}^{-1}$. Anal. calcd for $\mathrm{C}_{24} \mathrm{H}_{21} \mathrm{~N}_{3} \mathrm{OS}$ : C 72.15, H 5.30, N 10.52; found C 72.21, H 5.27, N 10.63.

T2: 棕色粉末状固体, $218.8 \mathrm{mg}$, 产率 42.1\%. m.p. $141 \sim 142{ }^{\circ} \mathrm{C}$; UV-Vis $\left(\mathrm{CHCl}_{3}\right) \lambda_{\max }: 258,292 \mathrm{~nm} ;{ }^{1} \mathrm{H}$ NMR $\left(400 \mathrm{MHz}, \mathrm{CDCl}_{3}\right) \delta: 7.00 \sim 8.27(\mathrm{~m}, 12 \mathrm{H}, \mathrm{ArH})$, $6.53(\mathrm{~s}, 1 \mathrm{H}, 2-\mathrm{H}), 4.58$ (d, $\left.J_{5 \mathrm{a}, 5 \mathrm{~b}}=15.2 \mathrm{~Hz}, 1 \mathrm{H}, 5-\mathrm{H}_{\mathrm{a}}\right), 3.37$ $\left(\mathrm{d}, J_{5 \mathrm{~b}, 5 \mathrm{a}}=15.2 \mathrm{~Hz}, 1 \mathrm{H}, 5-\mathrm{H}_{\mathrm{b}}\right), 3.14$ (q, $J=6.8 \mathrm{~Hz}, 4 \mathrm{H}$, $\mathrm{NCH}_{2} \mathrm{CH}_{3}$ ), 0.99 (t, $\left.J=7.2 \mathrm{~Hz}, 6 \mathrm{H}, \mathrm{NCH}_{2} \mathrm{CH}_{3}\right)$; IR (KBr) v: $3050(\mathrm{Ar}-\mathrm{H}), 2967(\mathrm{C}-\mathrm{H}), 1709(\mathrm{C}=\mathrm{O}), 1602(\mathrm{C}=$ $\mathrm{N}), 1592,1491(\mathrm{C}=\mathrm{C}), 1367(\mathrm{C}-\mathrm{N}), 1166,1049,687$ $(\mathrm{C}-\mathrm{S}-\mathrm{C}) \mathrm{cm}^{-1}$. Anal. calcd for $\mathrm{C}_{26} \mathrm{H}_{25} \mathrm{~N}_{3} \mathrm{OS}$ : C 73.04, $\mathrm{H}$ 5.89, N 9.83; found C 73.12, H 5.87, N 9.77.

T3: 棕色粉末状固体, $108.5 \mathrm{mg}$, 产率 $36.4 \%$. m.p. 230 $231{ }^{\circ} \mathrm{C}$; UV-Vis $\left(\mathrm{CHCl}_{3}\right) \lambda_{\max }: 245,290 \mathrm{~nm} ;{ }^{1} \mathrm{H}$ MNR (400 MHz, $\left.\mathrm{CD}_{3} \mathrm{OD}\right) \delta: 7.04 \sim 8.30(\mathrm{~m}, 12 \mathrm{H}, \mathrm{ArH})$, $6.31(\mathrm{~s}, 1 \mathrm{H}, 2-\mathrm{H}), 3.93(\mathrm{~s}, 1 \mathrm{H}, \mathrm{OH}), 4.38\left(\mathrm{~d}, J_{5 \mathrm{a}, 5 \mathrm{~b}}=15.4\right.$ $\left.\mathrm{Hz}, 1 \mathrm{H}, 5-\mathrm{H}_{\mathrm{a}}\right), 3.47\left(\mathrm{~d}, J_{5 \mathrm{~b}, 5 \mathrm{a}}=15.4 \mathrm{~Hz}, 1 \mathrm{H}, 5-\mathrm{H}_{\mathrm{b}}\right)$; IR
(KBr) v: $3371(\mathrm{O}-\mathrm{H}), 3076(\mathrm{Ar}-\mathrm{H}), 2925,2854(\mathrm{C}-\mathrm{H})$, $1703(\mathrm{C}=\mathrm{O}), 1611(\mathrm{C}=\mathrm{N}), 1560,1440(\mathrm{C}=\mathrm{C}), 1358$ $(\mathrm{C}-\mathrm{N}), 1163,1074,674(\mathrm{C}-\mathrm{S}-\mathrm{C}) \mathrm{cm}^{-1}$. Anal. calcd for $\mathrm{C}_{22} \mathrm{H}_{16} \mathrm{~N}_{2} \mathrm{O}_{2} \mathrm{~S}$ : C 70.95, $\mathrm{H} 4.33, \mathrm{~N} 7.52$; found $\mathrm{C} 71.02, \mathrm{H}$ $4.37, \mathrm{~N} 7.57$.

致谢 本文中对人白血病细胞生长的抑制率测试和体 外抗肿瘤活性测试由上海国家新药笁选中心完成.

\section{References}

[1] Kaluza, Z.; Abramski, W.; Chemielewski, M. Indian J. Chem. 1994, 338, 913.

[2] Imada, A.; Kitano, K.; Kintaka, K.; Muroi, M.; Asai, M. Nature 1981, 289, 590.

[3] Palomo, C.; Aizpurua, J. M.; Galarza, R.; Mielgo, A. J. Chem. Soc., Chem. Commun. 1996, 633.

[4] Verma, A.; Saraf, S. K. Eur. J. Med. Chem. 2008, 43, 897.

[5] Hamama, W. S.; Ismail, M. A.; Shaaban, S.; Zoorob, H. H. J. Heterocycl. Chem. 2008. 45, 939.

[6] Mosher, M. D.; Natale, N. R. J. Heterocycl. Chem. 1995, 32, 779.

[7] Phanstiel IV, O.; Price, H. L.; Wang, L.; Juusola, J.; Kline, M.; Shah, S. M. J. Org. Chem. 2000, 65, 5590.

[8] Chen, Q.-P.; Deady, L. W.; Baguley, B. C.; Denny, W. A. J. Med. Chem. 1994, 37, 593.

[9] Zhu, Y.-W.; Zhao, G.-C.; Zhou, Y.-Y. Chin. J. Synth. Chem. 2002, 10(1), 65 (in Chinese). (朱燕舞, 赵光超, 周运友, 合成化学, 2002, 10(1), 65.)

[10] Pihlaja, K.; Tahtinen, P.; Shaikhutdinov, R.; Hartikainen, H.; Ovcharenko, V.; Agirbas, H.; Guner, S. J. Heterocycl. Chem. 2004, $41,741$.

[11] Mollet, K.; D'hooghe, M.; Kimpe, N. D. Tetrahedron 2012, 68: 10787.

[12] Krasodomska, M.; Serda, P. Monatsh. Chem. 2007, 138, 199.

[13] Kumar, K. S.; Ganguly, S.; Veerasamy, R.; Clercq, E. D. Eur. J. Med. Chem. 2010, 45, 5474.

[14] Chhajed, S. S.; Manisha, P.; Bastikar, V. A.; Animeshchandra, H.; Ingle, V. N.; Upasani, C. D.; Wazalwar, S. S. Bioorg. Med. Chem. Lett. 2010, 20(12), 3640.

[15] Sharma, S.; Singh, T.; Mittal, R.; Saxena, K. K.; Srivastava1, V. K.; Kumar, A. Arch. Pharm. Chem. Life Sci. 2006, 339(3), 145.

[16] Popp, F. D. J. Org. Chem. 1962, 27, 2658.

[17] Tsuge, O.; Nishinohara, M.; Tashiro, M. Bull. Chem. Soc. Jpn. 1963, 36, 1477.

[18] Imerhasan, M.; Wang, T.; Helil, S.; Osman, K.; Muhammad, T. Chin. J. Org. Chem. 2010, 30(12), 1884 (in Chinese). (穆赫塔尔・伊米尔艾山, 王婷, 萨提瓦尔迪・海力力, 库尔班・吾 斯曼, 吐尔洪・买买提, 有机化学, 2010,30(12), 1884.) 